\title{
Pathological complete response of colorectal liver metastases following chemotherapy with S-1 and oxaliplatin (SOX) in combination with bevacizumab: A case report
}

\author{
TOMOYA YOKOTA ${ }^{1}$, TSUYOSHI SANO ${ }^{2}$, YASUHIRO SHIMIZU ${ }^{2}$, DAISUKE TAKAHARI ${ }^{1}$, \\ YOSHIKI SENDA ${ }^{2}$, MASAHIRO SHIMURA ${ }^{2}$, TAKASHI URA ${ }^{1}$, KOHEI SHITARA ${ }^{1}$, \\ YUJI NIMURA $^{2}$, YASUSHI YATABE ${ }^{3}$ and KEI MURO ${ }^{1}$ \\ Departments of ${ }^{1}$ Clinical Oncology, ${ }^{2}$ Gastroenterological Surgery, and ${ }^{3}$ Pathology and \\ Molecular Diagnostics, Aichi Cancer Center Hospital, Nagoya, Japan
}

Received September 6, 2010; Accepted December 7, 2010

DOI: 10.3892/ol.2011.256

\begin{abstract}
Pathological complete response to systemic chemotherapy is associated with more favorable survival in patients with colorectal cancer liver metastases. We present a case of a 63-year-old man with multiple liver metastases from descending colon cancer. Following surgical resection of the primary tumor, the patient received systemic chemotherapy with S-1 and oxaliplatin in combination with bevacizumab. On achievement of a markedly favorable response to chemotherapy, surgical treatment of liver metastases was performed, and the liver tumors were successfully resected without any macroscopic residue. Histopathological analyses showed necrotic tissue in the complete absence of residual viable tumor cells. This is the first reported case of a patient with multiple liver metastases from descending colorectal cancer to achieve a pathological complete response following systemic chemotherapy with S-1 and oxaliplatin in combination with bevacizumab. This regimen is a systemic chemotherapy option to 'cure' liver metastasis from colorectal cancer.
\end{abstract}

\section{Introduction}

The FOLFOX regimen, which includes bolus/infusional 5-fluorouracil (5-FU) with folinic acid modulation and oxaliplatin, has become one of the most common first-line treatments for patients with metastatic colorectal cancer (mCRC) (1). Despite high response rates (RRs) to the FOLFOX regimen, a pathological complete response (pCR) of CRC liver metastases to systemic chemotherapy is rarely achieved. Benoist et al

Correspondence to: Dr Tomoya Yokota, Department of Clinical Oncology, Aichi Cancer Center Hospital, 1-1 Kanokoden, Chikusa-ku, Nagoya 464-8681, Japan

E-mail: tomoya.yokota@gmail.com

Key words: pathological complete response, colon cancer, liver metastasis, SOX, bevacizumab reported that persistent macroscopic or microscopic residual disease or early recurrence in situ was observed in $83 \%$ of liver metastases downsized to a CR on imaging by systemic chemotherapy (2).

Since treatment with infusional 5-FU has the shortcomings of increased inconvenience, cost and morbidity related to the use of a portable infusion pump and a central venous catheter, oral fluoropyrimidines were evaluated as alternatives to infusional 5-FU. S-1 is an orally active prodrug of 5-FU that contains tegafur, which is constantly metabolized to 5-FU, combined with the modulators, gimeracil and potassium oxonate (3). In Japan, a phase I/II study of oxaliplatin plus oral S-1 (SOX) showed promising efficacy with good tolerability in patients with untreated mCRC (4). The efficacy of this combination was superior to that reported for each drug as monotherapy $(5,6)$, with a RR of $50 \%$, median progression-free survival (PFS) of 196 days, and a 1-year survival rate of $78.6 \%$. The results suggest that tri-weekly treatment with the SOX regimen is an adequate substitute for FOLFOX and can be administered more readily since it does not require central vein access. A phase I study of SOX plus bevacizumab (BV) showed that the maximum tolerated dose (MTD) of S-1 is $25 \mathrm{mg} / \mathrm{m}^{2}$. Moreover, no increases in toxicities were observed when BV and oxaliplatin were combined (7). However, the impact of SOX plus BV on CRC liver metastasis remains unknown.

This is the first reported case of multiple CRC liver metastases that had a marked response to SOX plus BV and achieved a pCR following radical liver resection.

\section{Case report}

A 63-year-old man was referred to a prior hospital due to bloody stool. Colonoscopic examination revealed a severely stenotic lesion in the descending colon, although the patient did not complain of any clinical symptoms. Subsequent abdominal computed tomography (CT) scans showed a tumor occupying the descending colon with possible invasion into the small intestine, along with multiple liver tumors at segments (S) 4, S5, S6 and S8 (Fig. 1A-D) (8). All metastases with the exception of that in $\mathrm{S} 8$ were $>30 \mathrm{~mm}$ in diameter. Positron emission 
tomography with 18-fluorodeoxyglucose (FDG-PET)/CT fusion imaging confirmed no other distant metastatic lesions. To prevent ileus due to complete obstruction in the descending colon, descending colectomy with lymphadenectomy was performed. Since the tumor was not mobilized due to direct invasion into the mesenterium and jejunum at surgery, both the involved proximal jejunum and the mesenterium were resected.

Pathological examination of the surgical specimens revealed the presence of a poorly differentiated adenocarcinoma (Fig. 2A) with direct invasion into the serosa of the jejunum. Tumor cells metastasized to the regional lymph nodes, including the paracolic (10/10) and left colic lymph nodes (1/1). The patient was referred to our hospital at that point for further treatment of the liver metastases.

Systemic chemotherapy with SOX plus BV was initially administered in the neoadjuvant setting. Briefly, oxaliplatin $\left(130 \mathrm{mg} / \mathrm{m}^{2}\right)$ and BV $(7.5 \mathrm{mg} / \mathrm{kg})$ were administered intravenously on day 1 , and S-1 $\left(80 \mathrm{mg} / \mathrm{m}^{2} /\right.$ day $)$ was administered orally twice daily for 14 days with a 1-week rest. The regimen was repeated every 3 weeks. Although no serious adverse events were observed, the patient developed grade 2 proteinuria during the treatment, which required suspension of $\mathrm{BV}$ in cycles 5,6 and 8. The patient did not receive BV during cycle 9 to allow washout of $\mathrm{BV}$ prior to liver resection.

The patient was monitored regularly with $\mathrm{CT}$ of the chest to pelvis, and by monthly measurement of carcinoembryonic antigen (CEA) levels. Following the completion of two cycles of SOX plus BV, each of the liver metastases was markedly decreased in size on CT scans, considered to reflect a partial response to the chemotherapy. The tumors in S4 and S8 were undetectable on the CT scans after five cycles of SOX plus BV (Fig. 1E and G), and the tumors in S5 and S6 decreased to 65 and 59\% of their original size, respectively (Fig. $1 \mathrm{~F}$ and $\mathrm{H}$ ). No evidence of any de novo detectable metastatic lesions was noted. The CEA level was slightly elevated prior to chemotherapy $(9.5 \mathrm{ng} / \mathrm{ml})$ and rapidly decreased to a normal level $(2.7 \mathrm{ng} / \mathrm{ml})$ at the completion of the five cycles of SOX plus BV. Retention rate of ICG at 15 min $\left(\mathrm{ICGR}_{15}\right)$ was $23 \%$ after nine cycles of SOX plus BV.

On achievement of a markedly favorable response to chemotherapy, the patient opted to undergo surgical treatment of the liver metastasis after nine cycles of SOX plus BV. Partial resection of S4, S5, S6 and S8 of the liver and cholecystectomy were performed. No evidence of peritoneal dissemination was observed on visual inspection. The liver displayed fatty change, but not the dark features of 'blue liver.' All of the liver tumors were successfully resected without any macroscopic residues.

The resected tumors were yellowish nodules and relatively large: $6 \times 5 \mathrm{~mm}$ in $\mathrm{S} 4,15 \times 10 \mathrm{~mm}$ in $\mathrm{S} 5$, and $13 \times 9 \mathrm{~mm}$ in S6 (Fig. 3). No mass lesion was identified in the S8 liver specimen, even after the tissue was sectioned into 24 pieces at 5 -mm intervals. Each nodule was sampled and histologically analyzed. Microscopic examination of the tissue revealed necrotic tissue without any viable tumor cells. The necrotic nodule was surrounded by a collagen capsule and was mixed with xanthogranuloma, comprising cholesterin cleft and foamy cells with moderate infiltration of inflammatory cells (Fig. 2B and $\mathrm{C}$ ). Sinusoidal obstruction was not identified in the non-

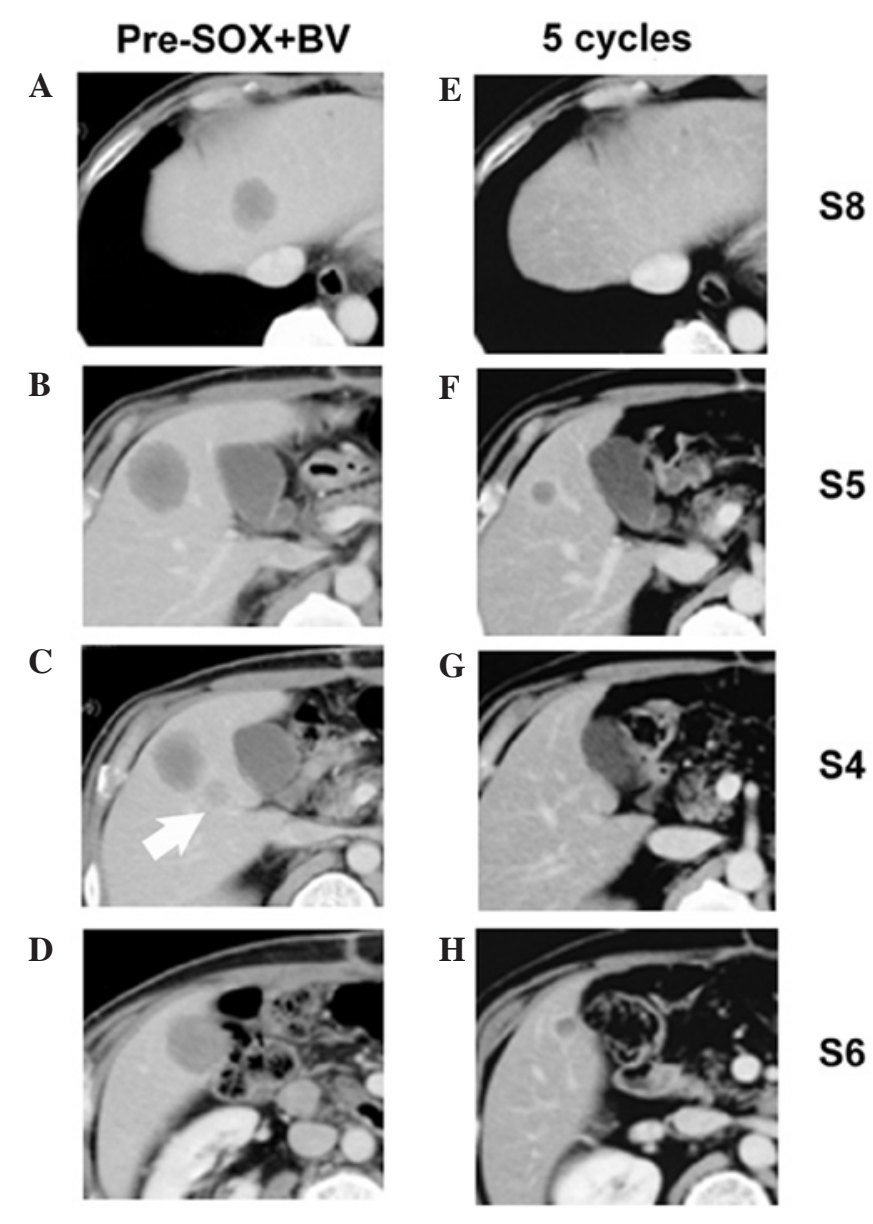

Figure 1. Abdominal computed tomography (CT) scan showing low-density areas in the liver prior to (A-D) and after (E-H) five cycles of SOX plus BV therapy. Arrow, solitary liver metastasis in S4.

cancerous liver tissue. Thus, a pCR was determined based on the absence of any viable tumor cells irrespective of the proportions of necrosis and fibrosis in any part of the resected liver tumor, corresponding to a grade 3 therapeutic effect.

Adjuvant chemotherapy with S-1 monotherapy is ongoing following liver resection. No radiological recurrence was observed 6 months following surgery.

\section{Discussion}

Patients with mCRC with synchronous liver metastases are known to have a poorer prognosis than patients with metachronous hepatic metastases (9-11). The potential occurrence of occult hepatic metastases should be considered in cases with synchronous liver metastases since primary colorectal cancer possesses a stronger effect than in cases with metachronous metastasis $(12,13)$. Survival rates of patients who do not undergo resection are also poor and do not exceed $2 \%$ at 5 years $(14,15)$. Previously, the introduction of novel chemotherapeutic agents, including oxaliplatin and irinotecan, increased the median survival of these patients $(1,16)$. However, outcomes of chemotherapy remain inferior to those of curative hepatic resection, resulting in 5-year survival rates of $40 \%$ overall (17) and even exceeding $50 \%$ in selected patients $(12,13,18,19)$. The 
A

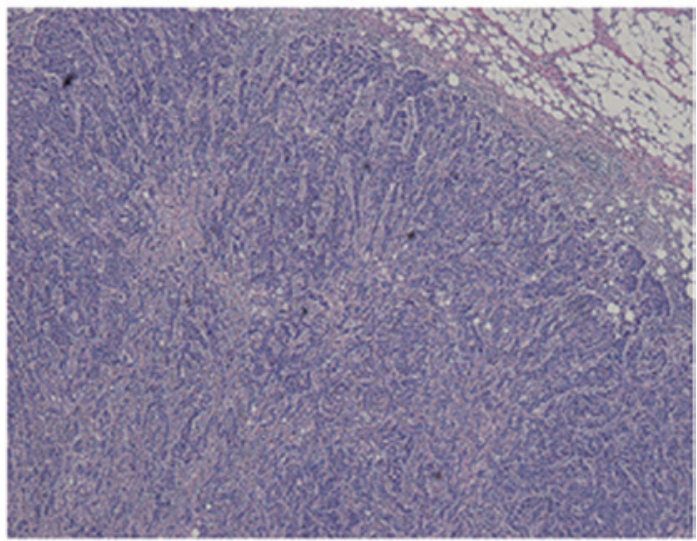

B

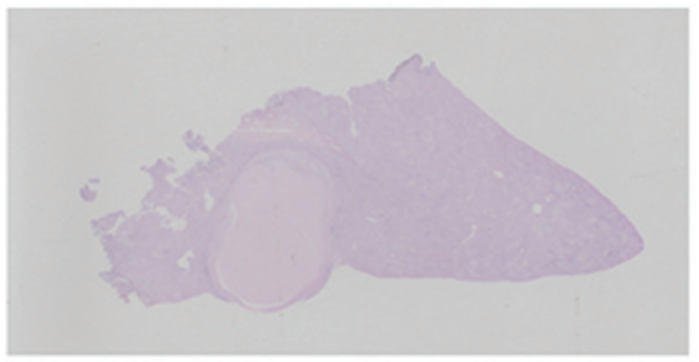

C

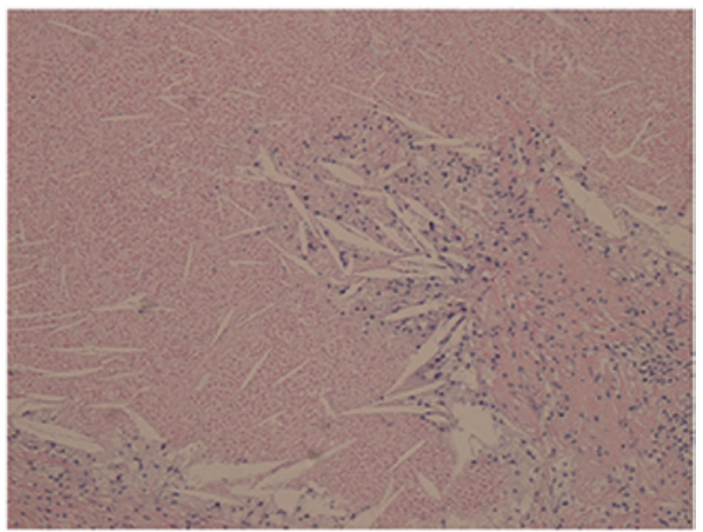

Figure 2. (A) A tissue sample from the primary descending colon tumor comprised diffuse infiltration of poorly differentiated adenocarcinoma. (B and C) At liver segment S6, the tumors histologically showed coagulation necrosis without any viable adenocarcinoma cells. (A and C) Original magnification, x100 and (B) loupe magnification. H\&E, hematoxylin and eosin.

present case had a locally advanced colon tumor with direct invasion into adjacent viscera and multiple metastatic regional lymph nodes. Pathological examination of the primary lesion revealed poorly differentiated adenocarcinoma. Multiple liver metastases were observed in the two lobes and were found to be synchronous. These findings suggested that the patient had a poor prognosis. Thus, a cure was unlikely to be achieved. Consequently, the Tumor Boards of our hospital recommended neoadjuvant chemotherapy, although multiple liver metastases in the two lobes were not technically unresectable.
S4

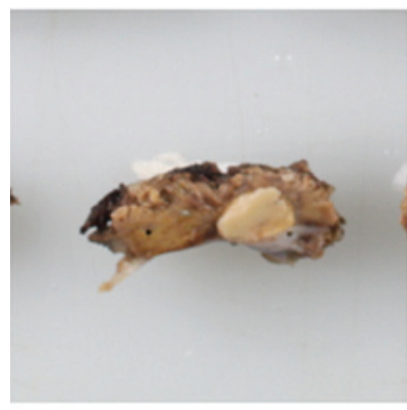

S6

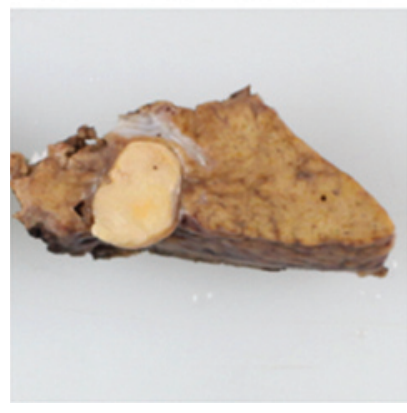

S5

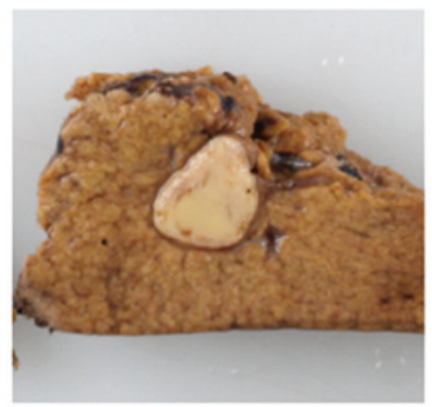

S8

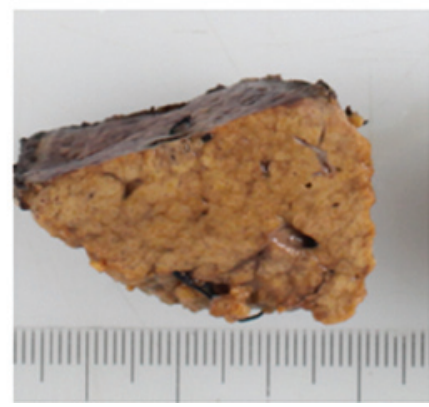

Figure 3. Macroscopic view of the cut surface of the resected liver. The tumor was homogeneous and yellowish-white in color.

Histopathological analysis of resected liver specimens potentially allows for the evaluation of tumor response following therapy. In particular, a pCR is defined as the complete absence of residual viable tumor cells on examination by pathologists as noted in the present case, and is associated with relatively high survival rates $(20,21)$. A multivariate analysis identified three variables as independent predictors of cure for patients with CRC liver metastases, including a maximum size of metastases at diagnosis of less than $30 \mathrm{~mm}$, three or fewer metastases at hepatectomy and a pCR (22). Thus, from an oncological perspective, a $\mathrm{PCR}$ appears to have greater clinical relevance compared to a radiologic $\mathrm{CR}$.

No correlation between radiological imaging and pathologic analysis was noted in this case. CT showed remnant liver lesions in S5 and S6 following chemotherapy, although a pCR was achieved. This suggests that the use of a single imaging modality, i.e., CT, is insufficient for the prediction of a pathological response in the evaluation of CRC liver lesions. Furthermore, a complete disappearance of liver lesions on radiological imaging does not always reflect a complete disappearance of viable tumor cells on pathological examination. Data suggest that sites of liver metastases with CR on imaging and without visible disease at surgery indicate the presence of viable tumor cells on pathological examination in $80 \%$ of patients (2). Therefore, it is optimal to remove any lesions containing a tumor, as performed in the present case. Furthermore, more accurate assessments of tumor cell viability are required during neoadjuvant chemotherapy. Adam et al reported that pCR was observed in $4 \%$ of patients with CRC 
liver metastases who received preoperative chemotherapy (20). Various reports proposed that the presence of metastases of $30 \mathrm{~mm}$ or less at diagnosis is a key predictive factor for pCR $(2,21)$. Although the maximum size of the metastases at diagnosis was more than $30 \mathrm{~mm}$ in the present case, a pCR was achieved.

In studies by Benoist et al (2) and Adam et al (20), the pathological response to chemotherapy was analyzed in CRC patients treated with 5-FU and leucovorin (LV5FU2), LV5FU2 plus oxaliplatin, and LV5FU2 plus irinotecan, but not LV5FU2 plus BV. The majority of pCR patients received FOLFOX without BV as the last line of systemic therapy prior to hepatectomy. pCR was observed in $6 \%$ of patients treated with FOLFOX and in $2 \%$ of patients who received FOLFIRI. No significant differences in the chemotherapy characteristics were noted between patients who did and did not experience a pCR (20). However, the efficacy of BV when combined with cytotoxic therapy in patients undergoing preoperative chemotherapy followed by liver resection remains unclear. For example, Ribero et al reported that the addition of BV to 5FU/oxaliplatin did not appear to increase the incidence of pCR (23). In contrast, a recent study showed that the rates of radical surgery are slightly higher in patients treated with an oxaliplatin-based regimen plus BV versus oxaliplatin-based chemotherapy alone (24). Furthermore, regimens including BV have been shown to be associated with a significantly higher frequency of complete or major responses compared to those without BV (25).

Long-term exposure to oxaliplatin is associated with an increased risk of postoperative liver insufficiency without improvement of pathological response (25-27). In the present case, a relatively high dose of total oxaliplatin $(1,930 \mathrm{mg})$ was administered, which may have been responsible for the high ICGR $_{15}$ prior to surgery. Therefore, hepatectomy for CRC metastases should be performed as early as possible, although there is no consensus regarding appropriate timing for hepatic resection. However, a retrospective study showed that oxaliplatin-related hepatic lesions occurred significantly less frequently in patients treated with oxaliplatin plus BV compared to those who received oxaliplatin alone $(23,28)$. This finding may support a model in which the preoperative use of BV in combination with SOX may contribute, not only to improvement of the pathological response, but also to a reduction of the risk of hepatotoxicity.

In the present case, adjuvant chemotherapy with S-1 monotherapy is ongoing after surgery. The S-1 regimen was selected for the reasons that: i) the patient was already treated with nine cycles of oxaliplatin-based therapy prior to surgery (although no persistent sensory neuropathy was observed), ii) no data support the efficacy of the addition of BV to adjuvant chemotherapy, and iii) no definite metastatic lesions were detected in other organs on post-operative CT.

In conclusion, this is the first patient with multiple CRC liver metastases who achieved a pCR following SOX plus BV combination chemotherapy. The SOX regimen is a safe and effective treatment for $\mathrm{mCRC}$ that does not require central vein access. The addition of BV to the chemotherapy may also have played a role in the $\mathrm{pCR}$. Therefore, systemic chemotherapy with SOX plus BV is promising as a conversion therapy for CRC liver metastasis unlikely to be 'cured'.

\section{References}

1. De Gramont A, Figer A, Seymour M, Homerin M, Hmissi A, Cassidy J, Boni C, Cortes-Funes H, Cervantes A, Freyer G, Papamichael D, Le Bail N, Louvet C, Hendler D, de Braud F, Wilson C, Morvan F and Bonetti A: Leucovorin and fluorouracil with or without oxaliplatin as first-line treatment in advanced colorectal cancer. J Clin Oncol 18: 2938-2947, 2000.

2. Benoist S, Brouquet A, Penna C, Julié C, El Hajjam M, Chagnon S, Mitry E, Rougier P and Nordlinger B: Complete response of colorectal liver metastases after chemotherapy: does it mean cure? J Clin Oncol 24: 3939-3945, 2006.

3. Kato T, Shimamoto Y, Uchida J, Ohshimo H, Abe M, Shirasaka T and Fukushima M: Possible regulation of 5-fluorouracil-induced neuro- and oral toxicities by two biochemical modulators consisting of S-1, a new oral formulation of 5-fluorouracil. Anticancer Res 21: 1705-1712, 2001.

4. Yamada Y, Tahara M, Miya T, Satoh T, Shirao K, Shimada Y, Ohtsu A, Sasaki Y and Tanigawara Y: Phase I/II study of oxaliplatin with oral S-1 as first-line therapy for patients with metastatic colorectal cancer. Br J Cancer 98: 1034-1038, 2008.

5. Ohtsu A, Baba H, Sakata Y, Mitachi Y, Horikoshi N, Sugimachi $\mathrm{K}$ and Taguchi T: Phase II study of S-1, a novel oral fluoropyrimidine derivative, in patients with metastatic colorectal carcinoma. Br J Cancer 83: 141-145, 2000.

6. Shirao K, Ohtsu A, Takada H, Mitachi Y, Hirakawa K, Horikoshi N, Okamura T, Hirata K, Saitoh S, Isomoto H and Satoh A: Phase II study of oral S-1 for treatment of metastatic colorectal carcinoma. Cancer 100: 2355-2361, 2004.

7. Zhang J, Chung K, Zergebel C, Urrea P, Quinones M and Saltz L: Phase I study of oral S-1 in combination with oxaliplatin (oxali) and bevacizumab (bev) in patients with advanced solid tumors. J Clin Oncol ASCO Annual Meeting Proceedings Part I. 25 (Suppl): 4091, 2007.

8. Couinaud C: Le foie. Etudes anatomiques et chirurgicales. Masson, Paris, 1957.

9. Beckurts KT, Holscher AH, Thorban S, Bollschweiler E and Siewert JR: Significance of lymph node involvement at the hepatic hilum in the resection of colorectal liver metastases. Br J Surg 84: 1081-1084, 1997.

10. Hananel N, Garzon J and Gordon PH: Hepatic resection for colorectal liver metastases. Am Surg 61: 444-447, 1995.

11. Scheele J, Stang R, Altendorf-Hofmann A and Paul M: Resection of colorectal liver metastases. World J Surg 19: 59-71, 1995.

12. Shimizu Y, Yasui K, Sano T, Hirai T, Kanemitsu Y, Komori K and Kato T: Treatment strategy for synchronous metastases of colorectal cancer: is hepatic resection after an observation interval appropriate? Langenbecks Arch Surg 392: 535-538, 2007.

13. Shimizu Y, Yasui K, Sano T, Hirai T, Kanemitsu Y, Komori K and Kato T: Validity of observation interval for synchronous hepatic metastases of colorectal cancer: changes in hepatic and extrahepatic metastatic foci. Langenbecks Arch Surg 393: 181-184. 2008.

14. Wood CB, Gillis CR and Blumgart LH: A retrospective study of the natural history of patients with liver metastases from colorectal cancer. Clin Oncol 2: 285-288. 1976.

15. Wagner JS, Adson MA, Van Heerden JA, Adson MH and Ilstrup DM: The natural history of hepatic metastases from colorectal cancer: A comparison with respective treatment. Ann Surg 199: 502-508, 1984.

16. Saltz LB, Cox JV, Blanke C, Rosen LS, Fehrenbacher L, Moore MJ, Maroun JA, Ackland SP, Locker PK, Pirotta N, Elfring GL and Miller LL: Irinotecan plus fluorouracil and leucovorin for metastatic colorectal cancer: Irinotecan Study Group. N Engl J Med 343: 905-914, 2000.

17. LiverMetSurvey: International registry of liver metastases of colorectal cancer, http://www.livermetsurvey.org.

18. Choti MA, Sitzmann JV, Tiburi MF, Sumetchotimetha W, Rangsin R, Schulick RD, Lillemoe KD, Yeo CJ and Cameron JL: Trends in long-term survival following liver resection for hepatic colorectal metastases. Ann Surg 235: 759-766, 2002.

19. Abdalla EK, Vauthey JN, Ellis LM, Ellis V, Pollock R, Broglio KR, Hess K and Curley SA: Recurrence and outcomes following hepatic resection, radiofrequency ablation, and combined resection/ablation for colorectal liver metastases. Ann Surg 239: 818-825, 2004. 
20. Adam R, Wicherts DA, de Haas RJ, Aloia T, Lévi F, Paule B, Guettier C, Kunstlinger F, Delvart V, Azoulay D and Castaing D: Complete pathologic response after preoperative chemotherapy for colorectal liver metastases: myth or reality? J Clin Oncol 26: 1635-1641, 2008

21. Blazer DG III, Kishi Y, Maru DM, Kopetz S, Chun YS, Overman MJ, Fogelman D, Eng C, Chang DZ, Wang H, Zorzi D, Ribero D, Ellis LM, Glover KY, Wolff RA, Curley SA, Abdalla EK and Vauthey JN: Pathologic response to preoperative chemotherapy: a new outcome end point after resection of hepatic colorectal metastases. J Clin Oncol 26: 5344-5351, 2008.

22. Adam R, Wicherts DA, de Haas RJ, Ciacio O, Lévi F, Paule B, Ducreux M, Azoulay D, Bismuth $\mathrm{H}$ and Castaing D: Patients with initially unresectable colorectal liver metastases: is there a possibility of cure? J Clin Oncol 27: 1829-1835, 2009.

23. Ribero D, Wang H, Donadon M, Zorzi D, Thomas MB, Eng C, Chang DZ, Curley SA, Abdalla EK, Ellis LM and Vauthey JN: Bevacizumab improves pathologic response and protects against hepatic injury in patients treated with oxaliplatin-based chemotherapy for colorectal liver metastases. Cancer 110: 2761-2767, 2007.

24. Okines A, Puerto OD, Cunningham D, Chau I, Van Cutsem E, Saltz L and Cassidy J: Surgery with curative-intent in patients treated with first-line chemotherapy plus bevacizumab for metastatic colorectal cancer First BEAT and the randomised phase-III NO16966 trial. Br J Cancer 101: 1033-1038, 2009.
25. Kishi Y,Zorzi D, Contreras CM, Maru DM, Kopetz S, Ribero D, Motta M, Ravarino N, Risio M, Curley SA, Abdalla EK, Capussotti L and Vauthey JN: Extended preoperative chemotherapy does not improve pathologic response and increases postoperative liver insufficiency after hepatic resection for colorectal liver metastases. Ann Surg Oncol: June 22, 2010 (Epub ahead of print).

26. Karoui M, Penna C, Amin-Hashem M, Mitry E, Benoist S, Franc B, Rougier P and Nordlinger B: Influence of preoperative chemotherapy on the risk of major hepatectomy for colorectal liver metastases. Ann Surg 243: 1-7, 2006.

27. Aloia T, Sebagh M, Plasse M, Karam V, Lévi F, Giacchetti S, Azoulay D, Bismuth H, Castaing D and Adam R: Liver histology and surgical outcomes after preoperative chemotherapy with fluorouracil plus oxaliplatin in colorectal cancer liver metastases. J Clin Oncol 24: 4983-4990, 2006.

28. Rubbia-Brandt L, Lauwers GY, Wang H, Majno PE, Tanabe K, Zhu AX, Brezault C, Soubrane O, Abdalla EK, Vauthey JN, Mentha $G$ and Terris B: Sinusoidal obstruction syndrome and nodular regenerative hyperplasia are frequent oxaliplatinassociated liver lesions and partially prevented by bevacizumab in patients with hepatic colorectal metastasis. Histopathology 56 : 430-439, 2010 